\title{
Kinetic performance and energy profile in a roller coaster electron transfer chain: a study of modified tetraheme-reaction center constructs
}

Jean Alric, Jérôme Lavergne, Fabrice Rappaport, André Verméglio, Katsumi Matsuura, Keizo Shimada, Kenji V.P. Nagashima

\section{Supplementary Information : Appendices A and B}

Appendix A. Matrix form of the kinetic equations. One state of the ET chain is specified by the distribution of electrons (symbolized here by the sign •) over the four redox centers, e.g. $\left(\mathrm{P} \bullet c_{559} c_{552^{\bullet}} c_{556}\right)$ or $\left(\mathrm{P} c_{559^{\bullet}} c_{552^{\bullet}} c_{556^{\bullet}}\right)$, etc. The number of electrons in the chain is conserved and we can consider separately the families of states with 1,2, 3 electrons. For the 4 states with a single $\bullet$, the system can be described by a vector $\mathbf{S}_{\mathbf{1}}$, where the successive rows indicate the fractions of the various states at time $t$ :

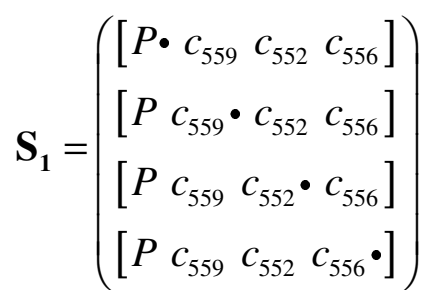

The system of ordinary differential equations that govern the time evolution of $\mathbf{S}_{\mathbf{1}}$ is:

$$
\frac{\mathrm{d} \mathbf{S}_{1}(\mathrm{t})}{\mathrm{dt}}=\mathbf{M}_{1} \mathbf{S}_{1}(\mathrm{t})
$$

with:

$$
\mathbf{M}_{1}=\left(\begin{array}{cccc}
-\mathrm{k}_{01} & \mathrm{k}_{10} & 0 & 0 \\
\mathrm{k}_{01} & -\mathrm{k}_{10}-\mathrm{k}_{12} & \mathrm{k}_{21} & 0 \\
0 & \mathrm{k}_{12} & -\mathrm{k}_{21}-\mathrm{k}_{23} & \mathrm{k}_{32} \\
0 & 0 & \mathrm{k}_{23} & -\mathrm{k}_{32}
\end{array}\right)
$$

For the two-electron family with 6 states, we write: 


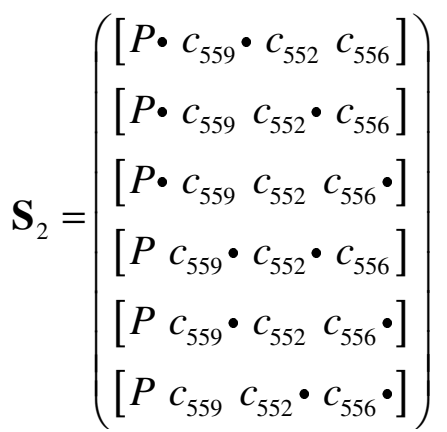

The corresponding evolution matrix is :

$$
\mathbf{M}_{2}=\left(\begin{array}{cccccc}
-\mathrm{k}_{12} & \mathrm{k}_{21} & 0 & 0 & 0 & 0 \\
\mathrm{k}_{12} & -\mathrm{k}_{21}-\mathrm{k}_{23}-\mathrm{k}_{01} & \mathrm{k}_{32} & \mathrm{k}_{10} & 0 & 0 \\
0 & \mathrm{k}_{23} & -\mathrm{k}_{32}-\mathrm{k}_{01} & 0 & \mathrm{k}_{10} & 0 \\
0 & \mathrm{k}_{01} & 0 & -\mathrm{k}_{01}-\mathrm{k}_{23} & \mathrm{k}_{32} & 0 \\
0 & 0 & \mathrm{k}_{01} & \mathrm{k}_{23} & -\mathrm{k}_{32}-\mathrm{k}_{12}-\mathrm{k}_{10} & \mathrm{k}_{21} \\
0 & 0 & 0 & 0 & \mathrm{k}_{12} & -\mathrm{k}_{21}
\end{array}\right)
$$

Finally, one has 4 states with 3 electrons described by:

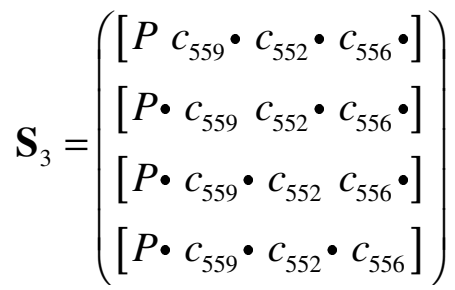

The evolution matrix is:

$$
\mathbf{M}_{3}=\left(\begin{array}{cccc}
-\mathrm{k}_{10} & \mathrm{k}_{01} & 0 & 0 \\
\mathrm{k}_{10} & -\mathrm{k}_{01}-\mathrm{k}_{21} & \mathrm{k}_{12} & 0 \\
0 & \mathrm{k}_{21} & -\mathrm{k}_{12}-\mathrm{k}_{32} & \mathrm{k}_{23} \\
0 & 0 & \mathrm{k}_{32} & -\mathrm{k}_{23}
\end{array}\right)
$$

Appendix B. A steady-state approximation for the slow phase. We consider the family of states with a single electron present. The kinetic scheme can be written:

$$
H_{3} \underset{k_{23}}{\stackrel{k_{32}}{\rightleftarrows}} H_{2} \underset{k_{12}}{\stackrel{k_{21}}{\rightleftarrows}} H_{1} \underset{k_{01}}{\stackrel{k_{10}}{\rightleftarrows}} P
$$

where states $P, H_{1}, H_{2}$ and $H_{3}$ and indicate that the electron is located on $\mathrm{P}, c_{559}, c_{552}$ or $c_{556}$, respectively (a shorthand notation for the lines of $\mathbf{S}_{\mathbf{1}}$, eq S1). We start with an electron in $c_{556}$ (state $\mathrm{H}_{3}$ ) and enquire about the kinetics of $\mathrm{P}^{+}$reduction (i.e. formation of state $P$ ). Obviously, 
the low potential heme $c_{552}$ will never reach a significant level of reduction (because the equilibrium constant $\mathrm{K}_{32}$ is about $1 / 400$ ) so that $\left[H_{2}\right]$ remains small and, except for the brief initial equilibration with $\mathrm{H}_{3}$, its derivative must be very small $\left(\mathrm{d}\left[\mathrm{H}_{2}\right] / \mathrm{dt} \approx 0\right)$ compared with that of states that undergo a significant overall evolution (e.g. $\mathrm{d}\left[H_{3}\right] / \mathrm{dt}$ ). Since there is no significant accumulation or depletion of $\mathrm{H}_{2}$, the net current $v$ is nearly the same from $\mathrm{H}_{3}$ to $\mathrm{H}_{2}$ and from $H_{2}$ to $H_{1}$ :

$v \approx k_{32}\left[H_{3}\right]-k_{23}\left[H_{2}\right] \approx k_{21}\left[H_{2}\right]-k_{12}\left[H_{1}\right]$

Solving these equations for $\left[H_{2}\right]$ and substituting, one obtains:

$v=k_{31}\left[H_{3}\right]-k_{13}\left[H_{1}\right]$

with:

$k_{31}=k_{32} \frac{k_{21}}{k_{21}+k_{23}} \quad k_{13}=k_{12} \frac{k_{23}}{k_{23}+k_{21}}=k_{31} \exp \left(\frac{\Delta G_{31}}{z}\right)=k_{31} / \mathrm{K}_{31}$

where $z=\mathrm{RT}$.

When $c_{559}$ is also at a low potential with respect to $c_{556}$ and $\mathrm{P}$, the same procedure can be applied one step further to obtain the overall ET rate from $c_{556}$ to P:

$k_{30}=\frac{k_{31} k_{10}}{k_{10}+k_{13}} \quad k_{03}=\frac{k_{30}}{K_{30}}$

where $K_{30}=\exp \left(\Delta \mathrm{G}_{30} / z\right)$ is the overall equilibrium constant. Since this constant is not very large (especially for the chimeric family), the relaxation rate of the $H_{3} \ldots P$ chain is not just $k_{30}$, but the sum of the forward and backward rate constants:

$k_{\text {slow }}^{a p}=k_{30}+k_{03}=k_{30}\left(1+\frac{1}{K_{30}}\right)$

Using eqs $S 11$ and S12 and rearranging, one can rewrite $k_{30}$ as:

$k_{30}=\left(\frac{1}{k_{32}}+\frac{1}{K_{32} k_{21}}+\frac{1}{K_{31} k_{10}}\right)^{-1}$

As commented in the paper, this form is instructive because it highlights the three possible 
modes of rate-limitation. The denominators in the three terms of eq S14 each indicate the maximum forward current that can be sustained across each step. For instance, on the $H_{1} \rightarrow P$ step, the rate is $k_{10}\left[H_{1}\right]$ and the maximum possible value for $\left[H_{1}\right]$ occurs when upstream equilibrium is satisfied. One has then:

$\left[H_{1}\right]=\frac{K_{31}}{1+K_{32}+K_{31}} \approx K_{31}$

where the approximation makes use of our starting assumption that both $c_{559}$ and $c_{552}$ have a low $\mathrm{E}_{\mathrm{m}}$ with respect to $c_{556}$ (thus, $K_{32}$ and $K_{31}<<1$ ). The condition of upstream equilibrium with respect to one kinetic step is precisely what is meant by the concept of rate-limitation on that step. The meaning of eq S14 is then clear: if the maximum possible current (denominator) for a given step is markedly smaller than for the others, then, (i) this step is rate-limiting, (ii) equilibrium is achieved in the section of the chain located upstream of this step and (iii) the overall rate equals the maximum rate that can be sustained at the rate-limiting step.

The preceding equations S11-S14 give valid effective rate constants whenever steadystate is achieved and could be generalized for a chain of any length. We argued that they can be applied safely for approximating $k_{\text {slow }}$ when the populations of $H_{2}$ and $H_{1}$ remain small because the midpoint potentials $E_{2}$ and $E_{1}$ are sufficiently low with respect to $E_{0}$ and $E_{3}$. This is clearly not true for the VC-F strain where $E_{1}>E_{0}$. Furthermore, the above treatment implied the presence of a single electron in the chain, whereas in the experiments with VC-F, two electrons are present, initially located on $c_{559}$ (we call this the "first electron") and $c_{556}$ ("second electron"). In this case, one can easily obtain a good approximation for the rate of the slow phase of $\mathrm{P}^{+}$reduction by noting that a rapid relaxation will first occur between $c_{559}$ and $\mathrm{P}$ for the first electron, meaning that it will then be present on $\mathrm{P}$ for a fraction $K_{10} /\left(1+K_{10}\right)$ of the time. On the other hand, eq S11 still applies for the second electron on the $H_{3} \ldots H_{1}$ segment when $c_{559}$ is oxidized. The effective overall forward rate constant should thus be: 
$k_{30}^{\prime} \approx k_{31} \frac{K_{10}}{1+K_{10}}$

and eq S13 remains valid using $k_{30}$ ' instead of $k_{30}$. Since in the region of high $E_{1}$ relevant to VC-F, the rate-limitation is on the first step $H_{3} \rightarrow H_{2}$, so that $k_{30}$ (from eq S12) $\approx k_{31} \approx k_{32}$ one may as well, for convenience, adopt a single expression for the slow phase, i.e.:

$k_{\text {slow }}^{a p}{ }^{\prime} \approx k_{\text {slow }}^{a p} \frac{K_{10}}{1+K_{10}}$

In the low $\mathrm{E}_{1}$ range, the second term (involving $\mathrm{K}_{10} \gg>1$ ) is close to 1, so that eq $\mathrm{S} 17$ becomes equivalent to the single electron expression $\mathrm{S} 13$; in the high $\mathrm{E}_{1}$ range, the first term is close to the more correct expression derived from eq S16. 